\title{
Vortex core structure and global properties of rapidly rotating Bose-Einstein condensates
}

\author{
Gordon Baym and C.J. Pethick \\ Department of Physics, University of Illinois at Urbana-Champaign, 1110 West Green Street, Urbana, Illinois 61801, \\ and \\ NORDITA, Blegdamsvej 17, DK-2100 Copenhagen Ø, Denmark
}

\begin{abstract}
We develop an approach for calculating stationary states of rotating Bose-Einstein condensates in harmonic traps which is applicable for arbitrary ratios of the rotation frequency to the transverse frequency of the trap $\omega_{\perp}$. Assuming the number of vortices to be large, we write the condensate wave function as the product of a function that describes the structure of individual vortices times an envelope function, varying slowly on the scale of the vortex spacing. By minimizing the energy, we derive Gross-Pitaevskii equations that determine the properties of individual vortices and the global structure of the cloud. For low rotation rates, the structure of a vortex is that of an isolated vortex in a uniform medium, while for rotation rates approaching the frequency of the trap (the mean field quantum Hall regime), the structure is that of the lowest p-wave state of a particle in a harmonic trap with frequency $\omega_{\perp}$. The global structure of the cloud is determined by minimizing the energy with respect to variations of the envelope function; for conditions appropriate to most experimental investigations to date, we predict that the transverse density profile of the cloud will be of the Thomas-Fermi form, rather than the Gaussian structure predicted on the assumption that the wave function consists only of components in the lowest Landau level.
\end{abstract}

\section{INTRODUCTION}

Bose-Einstein-condensed atomic gases are very well suited to investigating quantized vortex lines. Single vortex lines were first made in atomic condensates by Matthews et al. [1], who induced rotation by phase imprinting in a spinor condensate. Subsequently, arrays containing many vortices were created in scalar condensates by inducing rotation mechanically, either by stirring the condensate [2,3], or by evaporating particles [4]. For a theoretical review, see Ref. [5]. In a seminal work, Ho [6] predicted that clouds of atoms confined in harmonic traps, when rotated at frequencies close to the transverse frequency $\omega_{\perp}$ of the trap, should condense into the lowest Landau level (LLL) in the Coriolis force, similar to charged particles in the quantum Hall regime. This insight has led to extensive experimental studies in which rotation rates in excess of $0.99 \omega_{\perp}$ have been achieved, and the structure of the condensate within a single cell of the vortex lattice has been examined $[4,7]$.

To date, most theoretical work on vortices in harmonically trapped condensates rotating at frequencies close to $\omega_{\perp}$ has been based on the use of wave functions in which particles occupy only the lowest Landau level. In contrast, for slowly rotating condensates, the usual approach to calculating vortex structure is to solve the Gross-Pitaevskii equation. In this paper we address the question of how this approach goes over to the mean field quantum Hall description when the rotation rate is increased [8]. We develop a unified method for calculating both the structure of individual vortices and the global structure of the cloud for arbitrary rotation rates. Writing the condensate wave function as a product of a slowly varying envelope function that determines the density averaged over a single cell of the vortex lattice, and a function that determines the variations of the wave function on length scales of order the vortex separation and core size, we derive, in Sec. II, the energy of the system. Then in Sec. III we derive the equation for the structure of the wave function within a single cell of the vortex lattice by variation of the energy functional. In Sec. IV we derive equations for the global structure of the cloud. We find that a wave function made up only of components belonging to the lowest Landau level is a good approximation only under a very restricted range of conditions; if in the non-rotating system the density profile in the plane transverse to the rotation axis is of the Thomas-Fermi form, an inverted parabola, then at high rotation the shape remains Thomas-Fermi, and thus includes components from higher Landau levels, rather than the Gaussian shape predicted by the LLL calculation. 


\section{BASIC FORMALISM}

We consider a system of weakly interacting bosons trapped in a harmonic potential, $V(r)=\frac{1}{2} m\left(\omega^{2} r_{\perp}^{2}+\omega_{z} z^{2}\right)$, where $\vec{r}_{\perp}=(x, y)$, rotating at angular velocity $\Omega$ about the $z$ axis. The angular momentum of the system is carried in quantized vortices, of number $N_{v} \gg 1$ at large rotation rates. We assume the vortices to be rectilinear and to form a triangular lattice. When $N_{v} \gg 1$ the rotation rate of the lattice is related to the (two dimensional) density of vortices, $n_{v}$, by

$$
m \Omega=\pi \hbar n_{v}
$$

as follows from identifying the mean velocity via the condition for quantization of vorticity, $\oint_{\mathcal{C}} d \vec{\ell} \cdot \vec{v}=h N_{v}(\mathcal{C}) / m$, where $N_{v}(\mathcal{C})$ is the number of vortices surrounded by the contour $\mathcal{C}$. We will not address here the form of the corrections to Eq. (1) for finite $N_{v}[9]$.

In order to separate out the short distance vortex structure from the large scale structure, we follow the approach of Fischer and Baym [10] and write the order parameter as

$$
\psi(\vec{r})=e^{i \Phi(\vec{r})} f(\vec{r}) \sqrt{n(\vec{r})}
$$

the product of a rapidly varying real factor, $f(\vec{r})$, which vanishes at each vortex core, times a slowly varying real envelope function, $\sqrt{n(\vec{r})}$, and a phase factor. We normalize $f^{2}$ so that it averages to unity over each unit cell of the lattice; thus $n(\vec{r})$ is the smoothed density profile of the system, which varies slowly over the unit cells of the vortex lattice. The factor $e^{i \Phi} f$ describes the local swirling of the fluid - with the phase $\Phi$ wrapping by $2 \pi$ around each vortex - together with the overall rotation of the vortex lattice at $\Omega$. We generally set $\hbar=1$.

The total energy of the system in the laboratory frame is

$$
E=\int d^{3} r\left\{\frac{\hbar^{2}}{2 m}|\nabla \psi|^{2}+V(r) n(r) f^{2}(r)+\frac{g}{2} n^{2}(r) f^{4}(r)\right\}
$$

where we assume a two body interaction described by an s-wave scattering length, $a_{s}$, with $g=4 \pi a_{s} \hbar^{2} / m$. With Eq. (2), the kinetic energy in the laboratory frame becomes

$$
\left.\int d^{3} r \frac{1}{2 m}|\nabla \psi|^{2} \equiv K=\int d^{3} r \frac{1}{2 m}\left\{(\nabla \sqrt{n})^{2}+(\nabla \Phi)^{2} n f^{2}+n(\nabla f)^{2}+\frac{1}{2} \nabla f^{2} \cdot \nabla n\right)\right\} .
$$

We integrate the final term by parts to give $-\frac{1}{2} \int f^{2} \nabla^{2} n$; since $n$ varies slowly across a unit cell of the vortex lattice, we may replace the $f^{2}$ here by its average $(=1)$ in the cell, so that the integral gives only a vanishing surface term. Thus

$$
K=\int d^{3} r \frac{1}{2 m}\left\{(\nabla \sqrt{n})^{2}+(\nabla \Phi)^{2} n f^{2}+n(\nabla f)^{2}\right\}
$$

In the unit cell centered on vortex $j$ at position $\vec{R}_{j}$ in the plane transverse to the rotation axis, the velocity $\nabla \Phi / m$ is the sum of the solid body rotation of the position of the vortex, $\vec{\Omega} \times \vec{R}_{j}$, plus the local velocity around the vortex, which we write as $\nabla \phi_{j} / m$ :

$$
\nabla \Phi(r) \simeq m \vec{\Omega} \times \vec{R}_{j}+\nabla \phi_{j}
$$

The $(\nabla \Phi)^{2}$ term thus becomes

$$
\int d^{3} r \frac{n f^{2}}{2 m}(\nabla \Phi)^{2}=\sum_{j} \int_{j} d^{3} r n f^{2}\left\{\frac{\left(\nabla \phi_{j}\right)^{2}}{2 m}+\frac{1}{2} m \Omega^{2} R_{j}^{2}\right\}
$$

the integration is over unit cell $j$, and the sum is over all cells. The cross term vanishes since in the limit $N_{v} \gg 1$ the average velocity in the cell measured with respect to the center of the cell vanishes. In the Wigner-Seitz approximation, which we employ below, $\phi_{j}$ becomes the azimuthal angle measured with respect to the point $\vec{R}_{j}$. Writing within cell $j, \vec{R}_{j}=\vec{r}_{\perp}-\vec{\rho}$, the final term in Eq. (7) becomes $\frac{1}{2} I \Omega^{2}-\sum_{j} \int_{j} \frac{1}{2} m \Omega^{2} \rho^{2} n f^{2}$, where $I=\int d^{3} r m n f^{2} r_{\perp}^{2}$ is the total moment of inertia of the system. Similarly the transverse trapping potential term becomes $\frac{1}{2} I \omega^{2}$. 
To determine the equilibrium structure, we work in the frame rotating at angular velocity $\Omega$. [This procedure is equivalent to determining the equilibrium structure at fixed angular momentum, $L$, by minimizing the total energy taking the constraint of fixed $L$ into account by a Lagrange multiplier, $\Omega$.] The total angular momentum along the $z$ axis is given by

$$
L=\int d^{3} r n(r) f^{2}(r)(\vec{r} \times \nabla \Phi(r))_{z}
$$

Using Eq. (6), and again writing in cell $j, \vec{r}_{\perp}=\vec{R}_{j}+\vec{\rho}$, we have,

$$
L=I \Omega+\sum_{j} \int d^{3} r n f^{2}\left\{\left(\vec{\rho} \times \nabla \phi_{j}\right)_{z}-m \Omega \rho^{2}\right\}
$$

since the average position in the unit cell $j$ is $R_{j}$, and the average velocity in the local frame of the vortex $j$ vanishes. The first term is the angular momentum of the center of mass of the cell, and the second the intrinsic angular momentum within the cell. Assembling the pieces, Eqs. (3), (5), (7), and (9), we have,

$$
\begin{aligned}
E^{\prime}=E-\Omega L= & \int d^{3} r\left(\frac{(\nabla \sqrt{n})^{2}}{2 m}+\frac{m}{2} n(r) \omega_{z}^{2} z^{2}\right)+\frac{1}{2}\left(\omega^{2}-\Omega^{2}\right) I \\
& +\sum_{j} \int_{j} d^{3} r n\left[\frac{(\nabla f)^{2}}{2 m}+\frac{f^{2}}{2 m}\left(\nabla \phi_{j}\right)^{2}+\frac{m \Omega^{2}}{2} \rho^{2} f^{2} n-\Omega\left(\vec{\rho} \times \nabla \phi_{j}\right)_{z}+\frac{g}{2} n f^{4}\right] .
\end{aligned}
$$

Expressing $I$ in terms of the moment of inertia, $\bar{I}=\int d^{3} r m n r_{\perp}^{2}$, of the smoothed density distribution we write

$$
E^{\prime}=\int d^{3} r\left(\frac{(\nabla \sqrt{n})^{2}}{2 m}+\frac{m}{2} n(r) \omega_{z}^{2} z^{2}\right)+\frac{1}{2}\left(\omega_{\perp}^{2}-\Omega^{2}\right) \bar{I}+\sum_{j} E_{j}
$$

where

$$
E_{j}=\int_{j} d^{3} r n\left\{\frac{(\nabla f)^{2}}{2 m}+\frac{f^{2}}{2 m}\left(\nabla \phi_{j}\right)^{2}+\frac{m \omega_{\perp}^{2}}{2} \rho^{2}\left(f^{2}-1\right)+\frac{1}{2} m \Omega^{2} \rho^{2}-\Omega\left(\vec{\rho} \times \nabla \phi_{j}\right)_{z}+\frac{g}{2} n f^{4}\right\}
$$

is the internal energy within cell $j$.

\section{EQUILIBRIUM STRUCTURE OF VORTICES}

We turn now to determining the structure of the vortices within the unit cells. To do this we introduce the WignerSeitz approximation to evaluate the vortex sum, replacing the hexagonal unit cell by a circle of radius $\ell=1 /(m \Omega)^{1 / 2}$. Then $f$ is cylindrically symmetric within each cell. In the following, we assume that the vortex spacing is small compared with the characteristic length scale in the axial direction. The term in Eq. (12) containing $\partial f / \partial z$ can then be neglected, and $f$ depends only on the transverse coordinate and the average local density. In cell $j, \phi_{j}$ becomes the azimuthal angle with respect to the center of the cell. Again we write within cell $j, \vec{r}_{\perp}=\vec{R}_{j}+\vec{\rho}$, so that $\left(\nabla \phi_{j}\right)^{2}=1 / \rho^{2}$. Furthermore, $\left(\vec{\rho} \times \nabla \phi_{j}\right)_{z}$ becomes just $\hbar$, so that the angular momentum in the Wigner-Seitz approximation is,

$$
L=I \Omega+\sum_{j} \int_{j} d^{3} r n f^{2}\left(1-m \Omega \rho^{2}\right)=I \Omega+N\left(1-\frac{\left\langle\rho^{2}\right\rangle}{\ell^{2}}\right),
$$

where $\left\langle\rho^{2}\right\rangle \simeq \ell^{2} / 2$ is the average of $\rho^{2}$ within a given cell. We have neglected gradients of the smoothed density here. For an incompressible fluid, $\left\langle\rho^{2}\right\rangle / \ell^{2}=1 / 2$, and therefore the additional angular momentum per particle in the Wigner-Seitz approximation is $\hbar / 2$, which is close to Tkachenko's result $[11,12]$ for a triangular lattice in an incompressible fluid, $(\pi / 4 \sqrt{3}) \hbar \simeq 0.453 \hbar$.

In the Wigner-Seitz approximation, $E_{j}$ becomes,

$$
E_{j}=\int_{j} d^{3} r n\left\{\frac{1}{2 m}\left[\left(\frac{\partial f}{\partial \rho}\right)^{2}+\frac{f^{2}}{\rho^{2}}\right]+\frac{m}{2} \omega_{\perp}^{2} \rho^{2}\left(f^{2}-1\right)+\frac{1}{2} m \Omega^{2} \rho^{2}-\Omega+\frac{g}{2} n f^{4}\right\} .
$$


The form of $f$ within each cell is determined by minimizing $E_{j}$ with respect to $f$, subject to $\int_{j} d^{2} \rho f^{2}=1$, with the boundary conditions that $f(0)=0$ and $\partial f / \partial \rho=0$ at $\rho=\ell$. Since there are no terms coupling $f$ at different values of $z$, the equilibrium $f$ depends on $z$ only through the dependence of the average density on $z$. Thus within a given cell, at given height $z$,

$$
\frac{1}{2 m}\left(-\frac{1}{\rho} \frac{\partial}{\partial \rho}\left(\rho \frac{\partial f}{\partial \rho}\right)+\frac{f}{\rho^{2}}\right)+\frac{m \omega_{\perp}^{2}}{2} \rho^{2} f+g n f^{3}=\mu_{\text {cell }}\left(n\left(R_{j}, z\right)\right) f .
$$

This equation describes the vortex structure for all values of parameters, provided that $N_{v}$ is large. Equations (10) and (15) generalize the result of Ref. [10] through inclusion of the $(\nabla \sqrt{n})^{2} / 2 m$ and $m \omega_{\perp}^{2} \rho^{2} f^{2} / 2$ terms. The $(\nabla \sqrt{n})^{2}$ term allows us to go beyond Thomas-Fermi, when this energy dominates the interaction term. In the limit $\Omega \gg \omega_{\perp}^{2} / 2 g n$, appropriate to the regime described in Ref. [10], the $\omega_{\perp}^{2}$ term in (15) can be neglected.

It is useful to define the averages over the unit cell,

$$
\begin{gathered}
a=\frac{1}{2} \ell^{2}\left\langle\left(\frac{\partial f}{\partial \rho}\right)^{2}+\frac{f^{2}}{\rho^{2}}\right\rangle, \\
a_{h}=\frac{1}{2 \ell^{2}}\left\langle\rho^{2}\left(f^{2}-1\right)\right\rangle
\end{gathered}
$$

and

$$
b=\left\langle f^{4}\right\rangle
$$

these quantities are dependent on the density within the cell. Then quite generally,

$$
E_{j}=\int_{j} d^{3} r n\left\{\Omega\left(a-\frac{3}{4}\right)+\frac{\omega_{\perp}^{2}}{\Omega} a_{h}+\frac{g n}{2} b\right\} .
$$

For slow rotation, the core structure is basically that of a single vortex [13], and is reasonably well approximated by $[14]$

$$
f \sim \frac{\rho}{\left(2 \xi_{0}^{2}+\rho^{2}\right)^{1 / 2}}
$$

where $\xi_{0}=\hbar / \sqrt{2 m g n}$ is the Gross-Pitaevskii healing length. The corresponding density within this approximation to $f$ is shown as curve $a$ in Fig. 1 for the particular value, $\xi_{0}=0.1 \ell$.

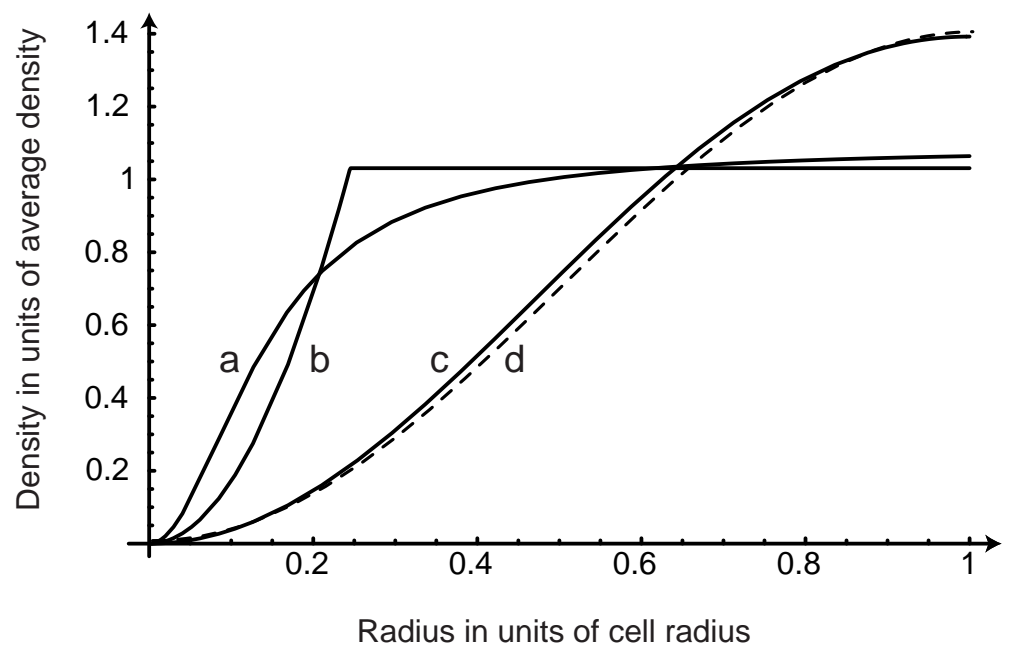

FIG. 1. Density within a vortex core in units of the average density in the cell, as a function of the transverse radius in units of the core radius $\ell$ : a) the single vortex form, $(20)$; b) the linear core approximation, (21); and c) the quantum Hall structure, $(29)$, d) the free particle Bessel function $J_{1}$ (dashed). Curves $a$ and $b$ are calculated for $\xi_{0}=0.1 \ell$. 
Reference [10] used a simple linear approximation for $f$ for all rotation speeds, in which $f$ rises linearly to the effective core radius $\xi$ and then becomes constant to the edge of the cell,

$$
f(\rho)=\frac{1}{\left(1-\xi^{2} / 2 \ell^{2}\right)^{1 / 2}} \times \begin{cases}\rho / \xi, & 0 \leq \rho \leq \xi \\ 1, & \xi \leq \rho \leq \ell .\end{cases}
$$

The corresponding density is shown as curve $b$ in Fig. 1 , for the value $\xi=\sqrt{6} \xi_{0}$ with $\xi_{0}=0.1 \ell$. In general one can solve Eq. (15) numerically for $s$, although we shall not do this here.

With the linear approximation (21), the individual vortex energy, $E_{j}$, becomes

$$
E_{j}=\int_{j} d^{3} r n\left(\Omega a(\zeta)+\frac{\omega_{\perp}^{2}}{\Omega} a_{h}(\zeta)+\frac{g n}{2} b(\zeta)-\frac{3 \Omega}{4}\right),
$$

where

$$
a(\zeta)=\frac{1-\frac{1}{2} \ln \zeta}{1-\zeta / 2}, \quad a_{h}(\zeta)=\frac{3 \zeta-2 \zeta^{2}}{12(2-\zeta)}
$$

and $\zeta=\xi^{2} / \ell^{2}$ is the fractional area occupied by the vortex core. The fluctuations in the density within a cell renormalize the (long wavelength) coupling constant [10] by a factor $\left.b=\left\langle n^{2}\right\rangle /\langle n\rangle^{2}\right\rangle$, given, for the ansatz (21), by

$$
b(\zeta)=\frac{1-2 \zeta / 3}{(1-\zeta / 2)^{2}} .
$$

The relative area occupied by the core at position $\left(r_{\perp}, z\right)$ is found by minimizing the integrand of $(22)$ at the density $n\left(r_{\perp}, z\right)$ :

$$
\frac{\partial}{\partial \zeta}\left(a(\zeta)+\frac{\omega_{\perp}^{2}}{\Omega^{2}} a_{h}(\zeta)\right)+\frac{g n}{2 \Omega} \frac{\partial}{\partial \zeta} b(\zeta)=0
$$

In the Thomas-Fermi regime, the sound velocity, $s$, in the center of the trap is given by

$$
m s^{2}=g b n(0)=\frac{\omega_{\perp}}{2}\left[\frac{15 N b a_{s}}{d_{\perp}} \frac{\omega_{z}}{\omega_{\perp}}\left(1-\frac{\Omega^{2}}{\omega_{\perp}^{2}}\right)\right]^{2 / 5}
$$

where $d_{\perp}=1 /\left(m \omega_{\perp}\right)^{1 / 2}$ is the oscillator length for transverse motion. We show, in Fig. 2, the corresponding prediction for $\zeta$ at the center of the trap as a function of rotational velocity for ${ }^{87} \mathrm{Rb}$, taking the representative values, $N=2.5 \times 10^{6}, \omega_{\perp} / 2 \pi=8.3 \mathrm{~Hz}$, and $\omega_{z} / 2 \pi=5.2 \mathrm{~Hz}$.

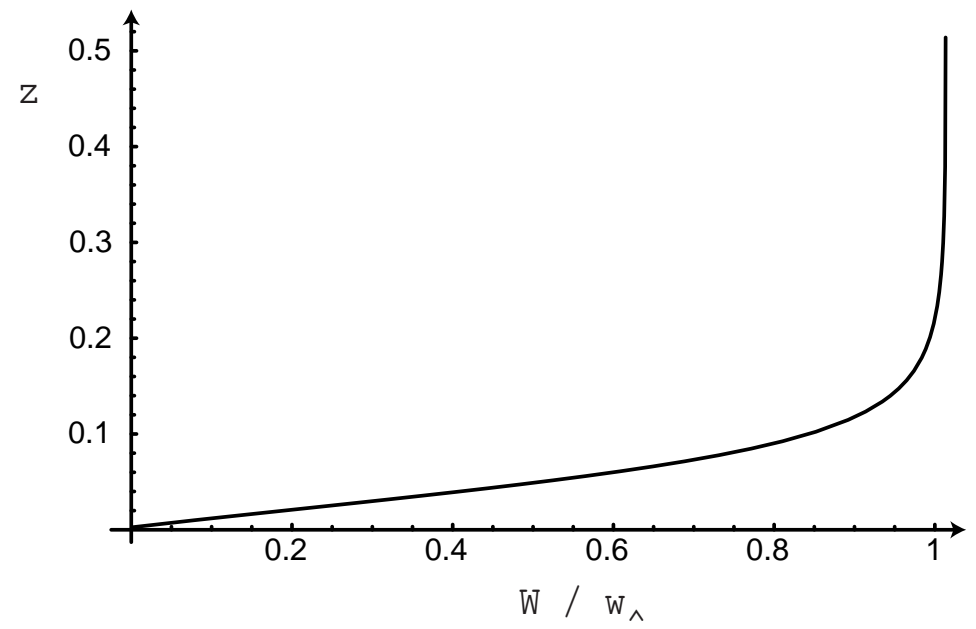

FIG. 2. Variation of the core size with rotational velocity, in the linear approximation to the core structure. 
As we see in Fig. 2, the core structure changes rapidly as $\Omega$ approaches the transverse trap frequency $\omega_{\perp}$. In order to study rotational velocities comparable to the transverse trapping frequency it is useful to spread out the horizontal scale by measuring rotational rates in terms of the rotational rapidity, $y$, defined by [15]:

$$
\frac{\Omega}{\omega_{\perp}} \equiv \tanh y
$$

or

$$
y=\frac{1}{2} \ln \frac{\omega_{\perp}+\Omega}{\omega_{\perp}-\Omega} .
$$

The rapidity variable essentially counts the number of 9 's in the fraction $\Omega / \omega_{\perp}$ as the fraction approaches unity (just as metal dealers describe the purity of metals). For example, the currently achieved $[7] \Omega / \omega_{\perp}=0.995$ corresponds to a rapidity of 3.00 , while $\Omega / \omega_{\perp}=0.999$ corresponds to $y=3.45$, and $\Omega / \omega_{\perp}=0.9999$ to $y=4.61$. In Fig. 3 we show the variation of the core size in Fig. 2, now as a function of rapidity.

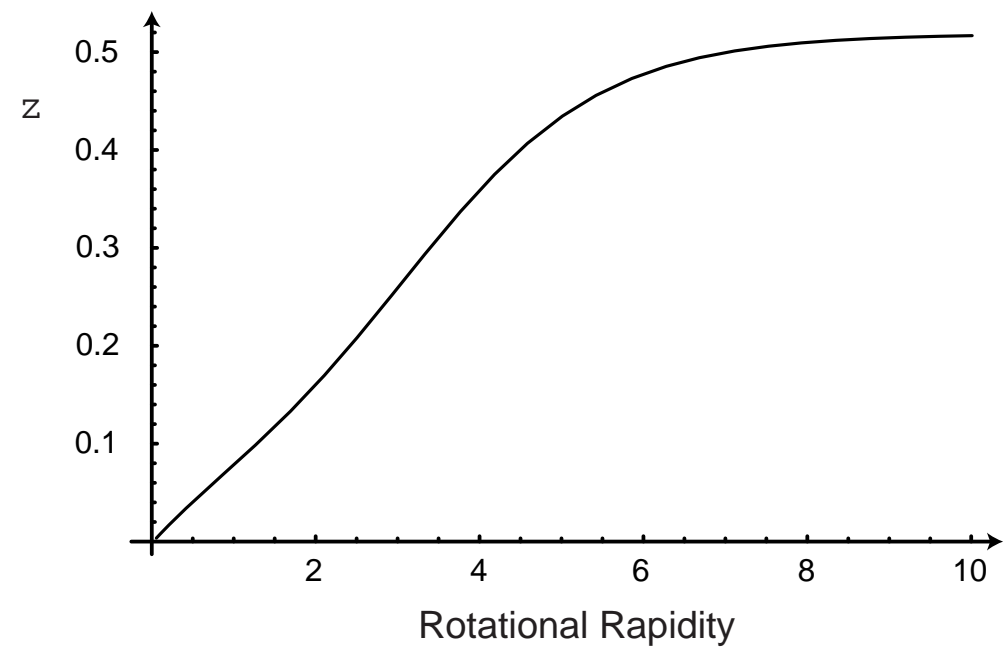

FIG. 3. Variation of the core size shown in Fig. 2, as a function of rotational rapidity, $y$.

In the mean field quantum Hall regime, where $\Omega$ approaches $\omega_{\perp}$, the cloud expands to the point where the gn term becomes a small perturbation on the structure within a cell; to lowest order $f$ assumes the particularly simple oscillator p-state structure,

$$
f=C \frac{\rho}{\ell} e^{-\rho^{2} / 2 \ell^{2}}
$$

plus small terms, where $C=(1-2 / e)^{-1 / 2}$. The p-wave solution for a particle in a trap with frequency $\Omega$ which satisfies the usual boundary condition $f \rightarrow 0$ for $\rho \rightarrow \infty$ kindly has zero slope precisely at $\rho=\ell$. With this form of $f$,

$$
E_{j}=\int_{j} d^{3} r n\left(\Omega+\frac{m}{2}\left(\omega_{\perp}^{2}-\Omega^{2}\right) \rho^{2}\left(f^{2}-1\right)+\frac{g}{2} n f^{4}\right),
$$

so that

$$
E^{\prime}=\int d^{3} r\left(\frac{(\nabla \sqrt{n})^{2}}{2 m}+\frac{m}{2} n \omega_{z}^{2} z^{2}+\frac{g n^{2} b}{2}\right)+N \Omega+\frac{1}{2}\left(\omega_{\perp}^{2}-\Omega^{2}\right) I
$$

this energy becomes the exact quantum Hall result in the limit, $\Omega \rightarrow \omega_{\perp}$. The average of $\rho^{2} / \ell^{2}$ is given by

$$
\frac{\int d^{2} \rho\left(\rho^{2} / \ell^{2}\right) f^{2}}{\int d^{2} \rho} \equiv b^{\prime}=\frac{2 e-5}{e-2}=0.608 ;
$$

in the linear core approximation one finds instead, 0.614. The renormalization of the coupling constant by fluctuations in the density within a cell, the factor $b=\left\langle n^{2}\right\rangle /\langle n\rangle^{2}$, is given in the quantum Hall limit by $[16,17]$, 


$$
b=\frac{\int d^{2} \rho f^{4}}{\int d^{2} \rho}=\frac{1}{4} \frac{e^{2}-5}{(e-2)^{2}}=1.158 ;
$$

by comparison, the linear core approximation yields 1.192. Note that the total moment of inertia, $I$, equals $\bar{I}+N\left(b^{\prime}-\right.$ $1 / 2) / \Omega$.

\section{GLOBAL STRUCTURE OF THE SYSTEM}

We turn now to determining the global structure of the cloud, by minimizing

$$
E^{\prime}=\int d^{3} r\left(\frac{(\nabla \sqrt{n})^{2}}{2 m}+\frac{m}{2} n \omega_{z}^{2} z^{2}\right)+\frac{1}{2}\left(\omega_{\perp}^{2}-\Omega^{2}\right) \bar{I}+\sum_{j} E_{j},
$$

at fixed particle number. From Eq. (34) we derive the effective Gross-Pitaevskii equation for the smoothed density:

$$
-\left\{\frac{\nabla^{2}}{2 m}+\frac{m}{2}\left(\omega_{z}^{2} z^{2}+\left(\omega_{\perp}^{2}-\Omega^{2}\right) r_{\perp}^{2}\right)+\mu_{\text {cell }}(n)\right\} \sqrt{n}=\mu \sqrt{n},
$$

where $\mu_{\text {cell }}=\delta E_{j} / \delta n$, defined by Eq. (15), contains the explicit interaction energy term gnb. In the quantum Hall limit, Eq. (30) implies that

$$
\mu_{\text {cell }}=2 \Omega+b g n+b^{\prime} \frac{\omega_{\perp}^{2}-\Omega^{2}}{2 \Omega} .
$$

The structure in the axial direction will, for $\Omega$ sufficiently close to $\omega_{\perp}$, always become Gaussian. The criterion for the axial structure to be Gaussian is that $g n$ be small compared with the axial oscillator frequency, $\omega_{z}$. Since the system density falls indefinitely with increasing $\Omega$, this condition will eventually be satisfied. From Eq. (26), the criterion is

$$
1-\frac{\Omega^{2}}{\omega_{\perp}^{2}} \ll\left(\frac{\omega_{z} b}{\omega_{\perp}}\right)^{3 / 2} \frac{2^{5 / 2} d_{\perp}}{15 N a_{s}}
$$

or in terms of rapidity,

$$
y \gg \frac{1}{2} \ln \left[\left(\frac{\omega_{\perp}}{\omega_{z}}\right)^{3 / 2} \frac{15 N a_{s}}{2^{1 / 2} d_{\perp}}\right] .
$$

For very weak interaction the transverse structure is Gaussian in the quantum Hall limit [6]:

$$
n\left(r_{\perp}, z\right)=\pi \sigma(z)^{2} e^{-r^{2} / \sigma(z)^{2}} \mathcal{N}(z),
$$

where $\mathcal{N}(z)$ is the number of particles per unit length in the axial direction. As we shall see below, such a Gaussian describes the system only for $N a_{s} \ll d_{z}$, where $d_{z}=1 /\left(m \omega_{z}\right)^{1 / 2}$ is the axial oscillator length. For this Gaussian,

$$
E^{\prime}=\int d z\left\{\frac{1}{2 m}\left(\frac{d(\mathcal{N}(z))^{1 / 2}}{d z}\right)^{2}+\mathcal{N}(z)\left(\frac{1}{2 m \sigma(z)^{2}}+\frac{m}{2}\left(\omega_{z}^{2} z^{2}+\left(\omega_{\perp}^{2}-\Omega^{2}\right) \sigma(z)^{2}\right)+\frac{b g}{4 \sigma(z)^{2}} \mathcal{N}(z)\right)\right\}
$$

plus a constant times $N$. Minimizing with respect to $\sigma(z)$ at fixed $\mathcal{N}(z)$, we find,

$$
\sigma(z)=d\left(1+2 \pi b \mathcal{N}(z) a_{s}\right)^{1 / 4}\left(\frac{\omega_{\perp}^{2}}{\omega_{\perp}^{2}-\Omega^{2}}\right)^{1 / 4}
$$

in agreement with Ref. [6].

However, if the transverse structure of the non-rotating cloud is Thomas-Fermi, it will remain Thomas-Fermi as the cloud is spun up, even to the quantum Hall limit. The criterion for the transverse structure to be Gaussian is different than in the axial direction, since the effective transverse oscillator frequency, $\left(\omega_{\perp}^{2}-\Omega^{2}\right)^{1 / 2}$, goes to zero. The criterion becomes instead that the interaction energy, $g n$, be small compared with the transverse kinetic energy: 
$g n \sim g N / Z R_{\perp}^{2} \ll 1 / 2 m R_{\perp}^{2}$, where $Z$ is the axial height and $R_{\perp}$ the transverse radius. This condition implies that $N a_{s} / Z$ be $\ll 1$. Since the total density per unit height, $N / Z$, increases with increasing $\Omega$ as the system flattens out, the structure in the transverse direction can only be Gaussian if the transverse structure in the non-rotating cloud is itself Gaussian. The maximum that $N / Z$ can become is $\sim N / d_{z}$, where $d_{z}$ is the axial oscillator length. For $N a_{s} / d_{z} \gg 1$ the structure in the radial direction will be Thomas-Fermi at large $\Omega$, even if it is Gaussian at small $\Omega$.

Note that in the quantum Hall limit, even though the interaction energy plays only a perturbative role in determining the structure within each cell of the lattice, it is crucial in determining the global structure. In particular, it is responsible for inclusion of components from higher Landau levels required to produce a Thomas-Fermi profile.

The final axial-Gaussian, transverse-Thomas-Fermi structure at high rotation has the form

$$
n(\vec{r})=e^{-z^{2} / d_{z}^{2}}\left(n(0)-\frac{m}{2 g b}\left(\omega_{\perp}^{2}-\Omega^{2}\right) r_{\perp}^{2}\right) .
$$

Using $\int d^{3} r n=N$, we find

$$
N=\frac{\pi^{3 / 2}}{2} d_{z} R_{\perp}^{2} n(0)
$$

where the transverse size, $R_{\perp}$, is given by the point where $n(\vec{r})$ falls to zero,

$$
R_{\perp}=\left(\frac{2 g b n(0)}{m\left(\omega_{\perp}^{2}-\Omega^{2}\right)}\right)^{1 / 2}=\left(8 \pi b a_{s} d^{2} n(0)\right)^{1 / 2}\left(\frac{\omega^{2}}{\omega_{\perp}^{2}-\Omega^{2}}\right)^{1 / 2}
$$

In terms of the total number, $N$,

$$
\frac{R_{\perp}}{d}=\frac{2}{\pi^{1 / 8}}\left(N b \frac{a_{s}}{d_{z}} \frac{\omega_{\perp}^{2}}{\omega^{2}-\Omega^{2}}\right)^{1 / 4}
$$

and

$$
n(0)=\frac{1}{2 \pi^{5 / 4}}\left(\frac{N}{b d^{4} d_{z} a_{s}} \frac{\omega^{2}-\Omega^{2}}{\omega_{\perp}^{2}}\right)^{1 / 2}
$$

\section{MEASURING THE CORE SIZE}

Several quantitative measures can be used to compare predicted core sizes with experiment, and with theory in the quantum Hall regime. The first is simply to compare the slopes of $f$ at the origin. The slope of the order parameter in the linear approximation, Eq. (21), is $1 / \ell(\zeta(1-\zeta / 2))^{1 / 2}$, which approaches $1.62 / \ell$ as $\Omega \rightarrow \omega_{\perp}$. On the other hand the quantum Hall wave function, $(29)$, has slope $1 / \ell(1-2 / e)^{1 / 2}=1.95 / \ell$. The second is to measure the mean square radius, $r_{c}^{2}$, of the density deficit in the core, defined by,

$$
r_{c}^{2}=\frac{\int_{j} d^{2} \rho\left[f(\ell)^{2}-f(\rho)^{2}\right] \rho^{2}}{\int_{j} d^{2} \rho\left[f(\ell)^{2}-f(\rho)^{2}\right]} .
$$

For the quantum Hall wave function, $r_{c}^{2} / \ell^{2}=((11 / 2)-2 e) /(3-e) \simeq 0.225$. To compare with the result from the linear approximation to $f$, we note that $r_{c}^{2} / \ell^{2}=\zeta / 3$, while as $\Omega$ approaches $\omega_{\perp}$, the value of $\zeta$ is found from the minimum of $a(\zeta)+a_{h}(\zeta)$, which is at $\zeta \simeq 0.519$; thus in this limit, $r_{c}^{2} / \ell^{2} \simeq 0.173$. Note that although the initial slope of the quantum Hall wave function is larger than that in the linear approximation, the mean square radius of the depression is also larger, since the depression in the quantum Hall wave function extends over the entire cell. Both measures of the core size in the linear approximation are in reasonable agreement with the exact quantum Hall result, given the simplicity of the approximation.

Experimentally, core properties are investigated after the rotating cloud has expanded. In the JILA experiments, the atoms are transferred to a state in which the magnetic forces tend to drive the cloud apart. It is therefore necessary to investigate how the vortex-core structure is affected by the transfer to the new state and the subsequent expansion of the cloud. Under expansion, the density drops, eventually reaching the point where the interaction 
energy no longer plays a role in determining the structure within the individual cells. The centrifugal force plays no role within a cell. If the potential is adiabatically turned off, allowing the system to expand slowly to the point where the interaction within a cell is small compared with the bending energy of the order parameter within a cell, or $1 / 2 m \ell^{2}=N_{v} / 2 m R_{\perp}^{2} \gg g N / R_{\perp}^{2} Z$. which is the case when the axial height expands to the point where $Z \gg 8 \pi a_{s} N / N_{v}$, then the structure within the individual cells, given by Eq. (15), is the Bessel function, $C_{1} J_{1}\left(x_{0} r / \ell^{\prime}\right)$, where $x_{0}=1.84$ is the location of the first maximum of the $J_{1}(x), C_{1}=2.05$ and $\ell^{\prime}$ is the cell size in the expanded cloud. In fact, the Bessel function solution is always within 0.015 of the quantum Hall solution, and the two solutions would be effectively indistinguishable in practice (see Fig. 1). The slope at the origin of the Bessel function is 1.88 , compared with 1.85 for the quantum Hall solution, while the mean square radius, $r_{c}^{2} / \ell^{2}$, of the depression is 0.231 , compared with 0.225 for the quantum Hall solution.

One can distinguish two stages in the evolution of the cloud during release and the subsequent expansion. The first is the period when the atoms are transferred to an untrapped state, and the second is expansion of the cloud in a modified trapping potential. The transfer of atoms occurs on a time scale short compared with dynamical times for the particles. Therefore the sudden approximation should be good, and changes in the coordinate-space wave function during the transfer should be negligible. This implies that both the global structure of the cloud and the structure of an individual cell of the vortex lattice are unchanged. After transfer of atoms to the new state, the structure within a cell will not correspond to the equilibrium configuration for the particular rotation rate because of the change in the trapping potential, which is determined by the instantaneous value of $\omega_{\perp}$. The calculations described in the previous paragraph demonstrate that when interaction effects are small, the structure of the condensate wave function within a single cell depends only weakly on $\omega_{\perp}$. Therefore, after transfer, the wave function within a cell will be the lowest state for the new value of $\omega_{\perp}$, apart from corrections of order one per cent. Likewise, for rotation rates so small that interaction effects dominate, we expect a similar conclusion to hold because the oscillator potential plays little role in determining the structure of an individual vortex.

We now consider the degree to which the vortex cores adjust adiabatically in the expansion. To do this, we compare the time scale, $\tau_{\text {cell }}$, for response of the structure of a cell of a vortex lattice with the expansion time scale, $\tau_{\exp }$. When the vortex core radius is small compared with the cell radius, the time for adjustments of the core is of order the core radius, $\sim(m g n)^{-1 / 2}$, divided by the sound speed $s$, or $\tau_{\text {cell }} \sim \hbar / g n$. When the core radius becomes comparable to the cell radius, i.e., $\hbar \Omega \gtrsim g n$, the inverse response time becomes of order the kinetic energy associated with a particle confined within a volume of radius $\ell$, divided by $\hbar$, or $\tau_{\text {cell }} \sim m \ell^{2} / \hbar=1 / \Omega$. Thus $1 / \tau_{\text {cell }}$ is always the larger of $g n / \hbar$ and $\Omega$. These estimates should apply at all stages in the evolution, provided that $n$ and $\Omega$ are the instantaneous values of these quantities. We note that if the expansion is purely two-dimensional, a good approximation for the recent experiments [7], the density and $\Omega$ both scale as $1 / R_{\perp}^{2}$; therefore, the ratio $g n / \hbar \Omega$ remains constant, and the core expansion rate always remains $g n / \hbar$ or $\Omega$.

There are similarly two regimes for the expansion. At low rotation rates, when the interaction energy per particle, $g n$, is large compared with $\hbar \omega_{\perp}$, the expansion velocity is determined by the interaction energy of the cloud, and is typically of order the sound velocity, $s_{0}$, in the cloud before release (the subscript 0 denotes quantities just prior to release). On the other hand, when the typical initial orbital velocity, $\Omega_{0} R_{\perp 0}$, exceeds the sound velocity, $s_{0}$, the dominant contribution to the expansion velocity after switching off the trap potential is the orbital motion, and therefore the expansion velocity is of order $\Omega_{0} R_{\perp 0}$. The typical expansion rate, $1 / \tau_{\exp }$, is thus always the larger of $s_{0} / R_{\perp}$ and $\Omega_{0} R_{\perp 0} / R_{\perp}$.

Now let us compare time scales. For low rotation velocities, $\Omega_{0} \lesssim s_{0} / R_{\perp 0}$, we have

$$
\frac{\tau_{\text {cell }}}{\tau_{\exp }} \sim \frac{1}{m s_{0} R_{\perp 0}} \frac{n_{0} R_{\perp 0}}{n R_{\perp}} \sim \frac{1}{m s_{0} R_{\perp 0}} \frac{R_{\perp}}{R_{\perp 0}},
$$

where the latter estimate holds for two dimensional expansion. This ratio is initially smaller than unity, implying that the cell initially adiabatically adjusts during the expansion, but if the cloud expands to a radius $\gtrsim R_{\perp 0}^{2} / \xi_{0}$, where $\xi_{0}$ is the Gross-Pitaevskii healing length, the condition for adiabaticity will be violated. For intermediate rotation rates, $s_{0} / R_{\perp 0} \lesssim \Omega_{0} \lesssim g n_{0} / \hbar$, the ratio of times is given by

$$
\frac{\tau_{\text {cell }}}{\tau_{\text {exp }}} \sim \frac{\hbar \Omega_{0}}{g n} \frac{R_{\perp 0}}{R_{\perp}} .
$$

This ratio starts at a value less than unity but increases $\propto R_{\perp} / R_{\perp 0}$ as the cloud expands. For the final case of fast rotation, $\Omega_{0} \gtrsim g n_{0} / \hbar$, the ratio is

$$
\frac{\tau_{\text {cell }}}{\tau_{\exp }} \sim \frac{\Omega_{0} R_{\perp 0}}{\Omega R_{\perp}} \sim \frac{R_{\perp}}{R_{\perp 0}} .
$$


In this case the adiabatic assumption is marginally satisfied initially, and is violated during the subsequent expansion. We conclude that one may draw no general conclusions about the development of vortex core structure during expansion on the basis of arguments about time scales; more detailed studies are needed.

It is interesting to note that states made up only of components in the lowest Landau level expand homologously when the effects of interaction are neglected [18]. In this case the structure of a single cell remains invariant, with only changes in scale, independent of the transverse length entering the wave function [cf. Eq. (29)]. Even though the condition for adiabaticity is violated, the structure of the single cell is precisely what would be predicted assuming adiabatic behavior.

\section{CONCLUSION}

In this paper we have developed a unified framework for describing the structure of rotating Bose-Einstein condensates containing a large number of vortices. We have derived a Gross-Pitaevskii equation which describes the structure of individual vortices and have demonstrated how the mean field quantum Hall state emerges as a simple continuation of the structure for small rotation rates. We find that the global density profile of the rotating clouds in the transverse direction is generally of the Thomas-Fermi form, rather than the Gaussian that emerges if only the lowest Landau level is occupied.

A number of open problems remain for future work. Throughout, we have assumed that the Gross-Pitaevskii approach may be used, and have neglected effects of excited states. One such effect is the zero-point motion of collective modes [21,17], which broadens the density profile of individual vortices and makes the lowest density nonzero. The density is the center of the vortex can also become non-zero via anomolous modes of excitation of the condensate [5] that in the linear approximation have a negative excitation energy. Even at zero temperature, such modes will have a non-zero equilibrium population such that the energy of an anomalous modes, including the effects of self-interaction, is just equal to zero. Explicit calculations are given in Ref. [19]. A third effect is the thermal population of excited states, which likewise will lead to a non-zero density at the center of the vortex. All of these effects must be taken into account in a detailed comparison of experiment with theory. Further problems include the quantitative delineation of the effect of expansion on the vortex core structure, and inclusion of effects of the lattice beyond the Wigner-Seitz approximation, such as the rigidity to shear motion, which manifests itself, e.g., in Tkachenko modes [11,20,21].

We thank James Anglin, Jason Ho, and Volker Schweikhard for many good discussions at the Aspen Center for Physics, to which we are grateful for giving us the opportunity to carry out this research. This work was supported in part by NSF Grant PHY00-98353.

[1] M.R. Matthews, B.P. Anderson, P.C. Haljan, D.S. Hall, C.E. Wieman, and E.A. Cornell, Phys. Rev. Lett. 83, 2498 (1999).

[2] K.W. Madison, F. Chevy, W. Wohlleben, and J. Dalibard,Phys. Rev. Lett. 84, 806 (2000); F. Chevy, K.W. Madison, and J. Dalibard, Phys. Rev. Lett. 85, 2223 (2000); K.W. Madison, F. Chevy, V. Bretin, and J. Dalibard, Phys. Rev. Lett. 86, $4443(2001)$.

[3] J.R. Abo-Shaeer, C. Raman, J.M. Vogels, and W. Ketterle, Science 292, 476 (2001).

[4] P.C. Haljan, I. Coddington, P. Engels, and E.A. Cornell, Phys. Rev. Lett. 87, 210403 (2001); P. Engels, I. Coddington, P.C. Haljan, and E.A. Cornell, Phys. Rev. Lett. 89, 100403 (2002).

[5] A.L. Fetter and A.A. Svidzinsky, J. Phys.: Condens. Matter 13, 135 (2001).

[6] T.-L. Ho, Phys. Rev. Lett. 87, 060403 (2001).

[7] I. Coddington, E. Cornell, P. Engels, and V. Schweikhard, private communication; V. Schweikhard, I. Coddington, P. Engels, V.P. Mogendorff, and E. Cornell, to be published.

[8] In this paper we confine ourselves to situations where the mean field approximation is valid, and will not deal with the interesting highly correlated states arising at very high rotation from quantum fluctuations. See, e.g., N.K. Wilkin, J.M.F. Gunn, and R.A. Smith, Phys. Rev. Lett. 80, 2265 (1998); N.K. Wilkin and J.M.F. Gunn, Phys. Rev. Lett. 84, 6 (2000); N.R. Cooper, N.K. Wilkin, J.M.F. Gunn, Phys. Rev. Lett. 87, 120405 (2001); S. Viefers, T.H. Hansson, and S.M. Reimann, Phys. Rev. A 62, 053604 (2000); N. Regnault and Th. Jolicoeur, cond-mat/0212477; and J.W. Reijnders, F.J.M. van Lankvelt, K. Schoutens, and N. Read, Phys. Rev. Lett. 89, 120401 (2002); cond-mat/0306402.

[9] When the number of vortices is finite, there is a net flow of fluid past the vortices. Thus $m \Omega / \pi$ will differ slightly from 
the vortex density, $N_{v} / \pi R_{\perp}^{2}$. The relative flow past the vortex cores produces a net Magnus force on individual vortices, of relative order $1-m R_{\perp}^{2} \Omega / N_{v}$, leading, e.g., to small distortions of the lattice from triangular.

[10] U. R. Fischer and G. Baym, Phys. Rev. Lett. 90, 140402 (2003).

[11] V.K. Tkachenko, Zh. Eksp. Teor. Fiz. 49, 1875 (1965) [Sov. Phys. JETP 22, 1282 (1966)]; Zh. Eksp. Teor. Fiz. 50, 1573 (1966) [Sov. Phys. JETP 23, 1049 (1966)].

[12] G. Baym and E. Chandler, J. Low Temp. Phys. 50, 57 (1983); 62, 119 (1986).

[13] L. Pitaevskii, Zh. Eksp. Teor. Fiz. 40, 646 (1961) [Sov. Phys. JETP 13, 451 (1961)].

[14] A. Fetter, in Lectures in Theoretical Physics, eds. K. Mahanthappa and W.E. Brittin, Vol. XIB, p. 351.

[15] We make this definition in direct analogy with the common concept of rapidity in high energy physics, $v / c=$ tanh $y$, where $v$ is the particle velocity and $c$ is the speed of light, introduced to distinguish speeds of ultrarelativistic particles.

[16] J. Sinova, C.B. Hanna, and A.H. MacDonald, Phys. Rev. Lett. 89, 030403 (2002).

[17] G. Baym, to be published.

[18] N. Read and N.R. Cooper, cond-mat/0306378.

[19] S. M. M. Virtanen, T. P. Simula, and M. M. Salomaa, Phys. Rev. Lett. 86, 2704 (2001) and J. Phys: Condens. Matter 13, L819 (2001).

[20] I. Coddington, P. Engels, V. Schweikhard, and E. Cornell, cond-mat/0305008.

[21] G. Baym, Phys. Rev. Lett. 2003 (in press), cond-mat/030529. 